\title{
Experimental-Theoretic Approach to Drug-Lymphocyte Interactome Networks with Flow Cytometry and Spectral Moments Perturbation Theory
}

\author{
Esvieta Tenorio-Borroto ${ }^{\mathrm{a}, \mathrm{b}}$, Fabiola Rivera Ramírez ${ }^{\mathrm{c}}$, Juan Carlos Vázquez Chagoyán ${ }^{\mathrm{a}}$, Roberto Montes de \\ Oca Jiménez ${ }^{\mathrm{a}}$, Xerardo García-Mera ${ }^{\mathrm{b}}$ and Humberto González-Díaz ${ }^{\mathrm{d}, \mathrm{e}}$,
}

\begin{abstract}
${ }^{a}$ Center for Research and Advanced Studies in Animal Health, Faculty of Veterinary Medicines and Animal Husbandry, Autonomous University of Mexico State (UAEM), Toluca, 50200, Estado de México, México; ${ }^{b}$ Department of Organic Chemistry, University of Santiago de Compostela (USC), 15782, Santiago de Compostela, Spain; ${ }^{c}$ Universidad Tecnológica del Valle de Toluca 52050 Santa maria de Atarrasquillo Lerma, México; ${ }^{2}$ Department of Organic Chemistry II, University of Basque Country UPV/EHU, 48940, Leioa, Spain; ${ }^{e} I K E R B A S Q U E$, Basque Foundation for Science, 48011, Bilbao, Spain
\end{abstract}

\begin{abstract}
We can combine experimental techniques like Flow Cytometry Analysis (FCA) with Chemoinformatics methods to predict the complex networks of interactions between organic compounds and targets in the immune system. In this work, we determined experimentally the values of $\mathrm{EC}_{50}=17.82 \mu \mathrm{g} / \mathrm{mL}$ and Cytotoxicity $=20.6 \%$ for the antimicrobial / anti-parasite drug Dermofural over Balb/C CD9 lymphocytes using flow cytometry. After that, we developed a new Perturbation-theory model for Drug-Cell Target Interactome in Lymphocytes based on dispersion-polarization moments of drug structure. The models correctly classifies 34591 out of 42715 (Accuracy $=80.9 \%$ ) cases of perturbations in assay endpoints of 11492 drugs (including both train and validation series). Each endpoint correspond to one out of 2616 assays, 38 molecular and cellular targets, 77 standard type measures, in four possible (human and rodents).
\end{abstract}

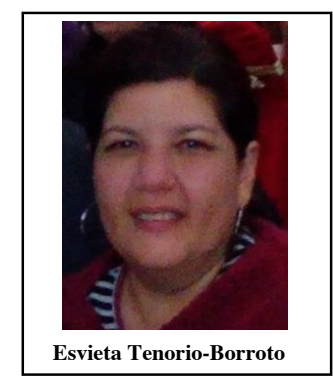

Keywords: Electronic charge, Atomic Polarizability, Dipole Moment, Spectral moments, Perturbation theory, Complex networks, ChEMBL, Drug immunotoxicity, Flow cytometry, CD19 Lymphocytes.

\section{INTRODUCTION}

We can combine multi-target networks models, perturbations theory, and flow cytometry to carry out a more complete study of the positive effects and/or cytotoxicity of drugs over different cell populations of the immune system. Specifically, Lymphocytes are also one of the more important cellular lineages of the immune system and play a central role in the immune response [1]. The main objective of the present work is to develop a valid Highthroughput mt-QSAR model for predicting the inmunotoxic effect of drugs over lymphocytes in a large set of $\mathrm{m}_{\mathrm{j}}$ assay conditions. Another important goal is to illustrate the use of the new method in a real-life example. Fort it, we are going to download and calculate TOPS-MODE selected descriptors for the large dataset reported in ChEMBL. Next, we shall fit and validate a new High-throughput mt-QSAR Linear Discriminant Analysis (LDA) model using the software STATISTICA. After that, we report, by the first time, the experimental study of the effect of the drug Dermofural over Balb/C mouse thymic lymphocytes $\mathrm{T}$ helpers (CD4+) and $\mathrm{T}$ cytotoxic $(\mathrm{CD} 8+)$ population using flow cytometry. Finally, we conduct the prediction of other endpoints multiplexing assay for Dermofural, not determined experimentally in this work. Also predicts the activity of some proteins that act in the immune response.

\section{REVIEW OF SPECTRAL MOMENTS ANALYSIS FOR MULTI-OUTPUT QSPR MODELING}

We can use different type of moments to quantify the structure of molecules in order to predict their interaction with complex

\footnotetext{
*Address correspondence to this author at the Department of Organic Chemistry II, University of the Basque Country (UPV/EHU), 48940, Bilbao, Spain; E-mail: humberto.gonzalezdiaz@ehu.es
}

soft-matter systems (proteins, cells, etc.) present in living organisms. The spectral moments $\left(\mu_{k}\right)$ of the atoms adjacency matrix $\mathbf{A}$ are very important for the definition of graph-spectrum-based molecular structural descriptors. In this context, the spectrum of the graph $\mathrm{G}$ is the set of the eigenvalues $\lambda_{i}$ of $\mathbf{A}$. The formula for the calculation of $\mu_{k}$ is the following:

$\mu_{\mathrm{k}}=\operatorname{Tr}\left(\mathbf{A}^{\mathrm{k}}\right)=\sum_{\mathrm{i}=0}^{\mathrm{n}} \lambda_{\mathrm{i}}{ }^{\mathrm{k}}$

Specifically, Babic, Graovac, and Gutman [2] reported a relationship for a descriptor called the energy $E(\mathrm{G})$ of a graph. $E(\mathrm{G})$ is based on the new concept of the resonance energy in conjugated hydrocarbons introduced by Jiang and Zhang [3]. The Jiang and Zhang [3] model involves an expansion of the $\pi$-electron energy in terms of spectral moments $\mu_{k}$, which are equal to numbers of closed walks in a molecular graph [4]. Babic, Graovac, and Gutman defined $E(\mathrm{G})$ as follows:

$\mathrm{E}(\mathrm{G})=\sum_{\mathrm{k}=0}^{\mathrm{n}} \lambda_{\mathrm{i}}$

Estrada et al. introduced the method TOSS-MODE [5, 6], and later renamed it as the TOPS-MODE method [7-9]. Both versions are useful to quantify the structure of molecules using the spectral moments of the bond (edge) adjacency matrix $\mathbf{B}\left(\mathrm{w}_{\mathrm{t}}\right)$ [5-9]. Estrada and Peña $[10,11]$ used this method to detect critical fragments for sedative/hypnotic compounds present in the Merck Index. Both $\mathbf{B}\left(\mathrm{w}_{\mathrm{t}}\right)$ and $\mu_{\mathrm{k}}\left(\mathrm{w}_{\mathrm{t}}\right)$ depends on bond weighting functions $\mathrm{w}_{\mathrm{t}}$; which in turn depend on atomic ${ }^{\mathrm{a}} \mathrm{w}_{\mathrm{t}}$ or ${ }^{\mathrm{b}} \mathrm{w}_{\mathrm{t}}$ bond physicochemical properties of type t. Atomic mass and bon standard distances are examples of different types of properties [7-9]. 


$$
\mu_{\mathrm{k}}\left(\mathrm{w}_{\mathrm{t}}\right)=\operatorname{Tr}\left[\mathbf{B}\left(\mathrm{w}_{\mathrm{t}}\right)^{\mathrm{k}}\right]
$$

In any case, almost all predictive studies based on numerical molecular descriptors of the chemical structure of organic compounds $\left(\mathrm{D}_{\mathrm{i}}\right)$ are unable to perform multi-target predictions. In fact, classic predictive methods for molecule-target interaction use only to predict the biological effect of single molecules over one target or system $[12,13]$. This situation applies also to almost all previous models based on spectral moments like $\mu_{\mathrm{k}}$ values.

However, new multi-target methods can predict properties of drugs, macromolecular structures, and macroscopic entities like populations taking into consideration changes in characteristics of the system. In doing so, González-Díaz et al. have used BoxJenkins moving average operators to describe multi-target situations [14-17]. These operators are the molecular version of the classic Moving Average (MA) component of the Box-Jenkins ARIMA models in time series [18]. We see these characteristics of the system as sets of physical and/or bio-molecular boundary conditions $\mathrm{c}_{j}$. Some of these boundary conditions may be $\mathrm{c}_{1}=$ drug bio-molecular targets, $c_{2}=$ species of organisms, $c_{3}=$ specific assay protocols, $c_{4}=$ temperature or time ranges, $\mathrm{c}_{5}=$ solvents, etc. We calculated the Box-Jenkins operators as the deviation of the molecular structure descriptor $D_{i}$ of one compound from the average value $\left\langle D_{i}\left(c_{j}\right)\right\rangle$. This is the average value for the structural descriptors of a sub-set of molecules measured under the same condition $\mathrm{c}_{\mathrm{j}}$.

$\Delta \mathrm{D}_{\mathrm{k}}={ }^{\mathrm{i}} \mathrm{D}_{\mathrm{k}}-\left\langle{ }^{\mathrm{i}} \mathrm{D}_{\mathrm{k}}\left(\mathrm{c}_{\mathrm{j}}\right)\right\rangle$

The method has a high potential for the study of complex biomolecular systems in the frontiers between Biophysics and Biochemistry. One interesting case appears when the molecular descriptors ${ }^{\mathrm{i}} \mathrm{D}_{\mathrm{k}}$ of type $\mathrm{k}^{\text {th }}$ for the $\mathrm{i}^{\text {th }}$ drug are the spectral moments of the adjacency matrix ${ }^{\mathrm{i}} \mathrm{D}_{\mathrm{k}}={ }^{\mathrm{i}} \mu_{\mathrm{k}}$. Very recently, Luan et al. [19] used Box-Jenkins operators of spectral moments to predict the interaction of chemical compounds with 148 possible molecular or cellular targets in 11 model organisms (including human). Cytotoxicity assays are screening methods that uses different permanent cell lines for ranking acute toxicities of parent compounds [20,21]. We can use different types of molecular parameters to predict the interaction of drugs with different targets in immune system. Tenorio et al. [22] also used spectral moments to find a multi-target model for drug immunotoxicity over 36 molecular or cellular targets in macrophages. In this particular case of spectral moments, we can write MA operators as follow:

$\Delta \mu_{\mathrm{k}}\left(\mathrm{w}_{\mathrm{t}}\right)={ }^{\mathrm{i}} \mu_{\mathrm{k}}\left(\mathrm{w}_{\mathrm{t}}\right)-\left\langle{ }^{\mathrm{i}} \mu_{\mathrm{k}}\left(\mathrm{w}_{\mathrm{t}}\right)\right\rangle_{\mathrm{j}}$

\section{EXPERIMENTAL STUDY OF THE EFFECT DERMO- FURAL OVER CD19 LYMPHOCYTE TARGETS}

The measurement of cell viability is an important goal in cytotoxicity studies [23-26]. In our study, we examined the populations of CD19 lymphocytes using flow cytometry. The analyses with flow cytometry were performed in order to follow the percentage of live lymphocytes present in the lymphocytes populations treated with Dermofural at different concentrations we observed changes in the viability of the lymphocytes after 24 hours. The assay shows a significant increase of dead cells, Cytotoxicity $(\%)=20.6 \%$, compared to the group untreated $(1.5 \%)$ and the DMSO group $(3.1 \%)$ at $c_{\max }=10 \mu \mathrm{g} / \mathrm{mL}$. The treatment of 6 and $8 \mu \mathrm{g} / \mathrm{mL}$ results in a dose-dependent significant increase in cytotoxicity $(14.5 \%)$ and $(17.4 \%)$ respectively (Fig. 1).

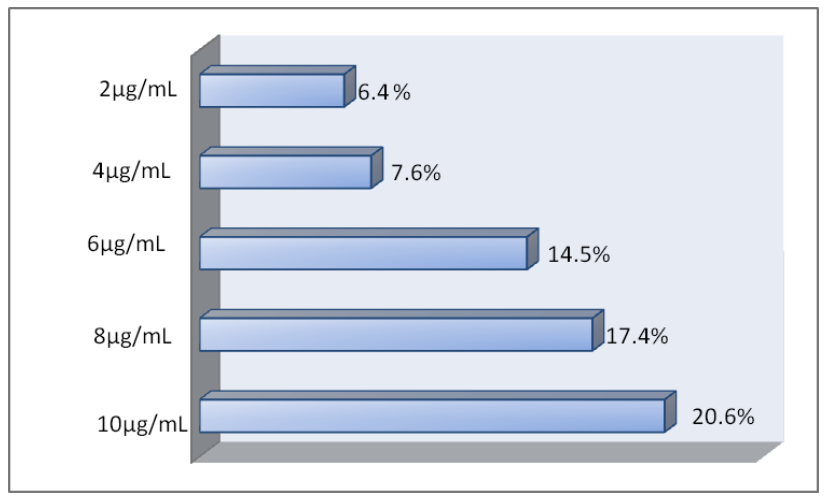

Fig. (1). Percentage of cytotoxicity in Balb/C mouse tymic B-Lymphocytes marked with CD19bioAPC/7AAD exposed to different concentrations of Dermofural.

The percent of cytotoxicity is similar in concentrations 2 and 4 $\mu \mathrm{g} / \mathrm{mL}$ (approximately 7\%). In studies [27] with these derivatives have worked with other ranges of cytotoxicity $(1.25$ to $30 \mu \mathrm{g} / \mathrm{ml}$.). These results indicate that has low cytotoxicity Dermofural at this concentration $(10 \mu \mathrm{g} / \mathrm{mL})$ for cytotoxicity $(\%)<50 \%$ is smaller than a threshold value is considered to cytotoxic compounds [28]. In general, there is no significant difference between different concentrations of DMSO group Dermofural. In Table 1, we show the results of statistical analysis of the parameters obtained of the flow cytometer, using the values for $\mathrm{p}$ and significant differences for $\mathrm{p}<$ 0.05 between different concentrations and control groups (Negative Control, DMSO), and then the average values of MFI in SSC and/or FSC scattering mode, for all samples. We confirm that there were significant differences for treated samples of living B lymphocytes with respect to control groups.

We also investigated the MFI on highly homogenous lymphocytes populations defined by the expression of CD19bioAPC obtained from the thymus of healthy mouse. In Fig. (2), we depict results for thymus B Lymphocytes of Balb/ C mouse analysis exposed to compound Dermofural at $10 \mu \mathrm{g} / \mathrm{mL}$. This corroborates that the B-lineage cells in the thymus account for less than $1 \%$ of total lymphocytes.

\section{PT-QSPR MODEL FOR DRUG-LYMPHOCYTE TARGET INTERACTOME}

We introduced one special case of QSPR-perturbation theory model combining the spectral moments of a molecular graph ${ }^{\mathrm{i}} \mu_{\mathrm{k}}\left(\mathrm{Q}_{\mathrm{t}}\right)$ and the approach to multiple input-output perturbations in biomolecular systems reported by Gonzalez-Díaz et al. [29, 30]. Please, see details about the method in these previous woks. Here we used this previous method to develop the first QSTRPerturbation model to predict interactions of organic compounds with molecular and cellular targets on lymphocyte cells (see Fig. 3). We downloaded the database from ChEML https://www.ebi.ac.uk/chembldb [31] with >20,000 assays of drugs related somehow to lymphocytes. The best QSPR-Perturbation model of first order found here with LDA was:

$$
\begin{aligned}
\mathrm{S}\left(\varepsilon_{\mathrm{ij}}\right)_{\mathrm{new}}= & 0.1578 \cdot \mathrm{f}\left(\varepsilon_{\mathrm{iv}_{\mathrm{ij}}}\right)_{\text {ref }}+33.5734 \cdot \Delta \mu\left(\mathrm{Q}_{0}\right)_{\mathrm{co}}+1.5541 \cdot \Delta \mu\left(\mathrm{Q}_{0}\right)_{\mathrm{cl}}-33.4696 \cdot \Delta \mu\left(\mathrm{Q}_{0}\right)_{\mathrm{c} 2} \\
& -34.4871 \cdot \Delta \mu\left(\mathrm{Q}_{1}\right)_{\mathrm{c} 0}+33.5374 \cdot \Delta \mu\left(\mathrm{Q}_{1}\right)_{\mathrm{c} 2}+33.5374 \cdot \Delta \Delta \mu(\mathrm{D})_{\mathrm{c} 2} \\
& -2.0914 \cdot \Delta \mu\left(\mathrm{Q}_{2}\right)_{\mathrm{cl}}+1.4713 \cdot \Delta \mu\left(\mathrm{Q}_{3}\right)_{\mathrm{cl}}-0.0693 \\
& \mathrm{~N}=42715 \quad \mathrm{~F}=2470.742 \quad \mathrm{U}=0.15 \quad \mathrm{p}<0.005
\end{aligned}
$$

The first input term $\mathrm{f}\left(\varepsilon_{\mathrm{ij}}\right)_{\text {ref }}$ is the scoring function $\mathrm{f}$ of the efficiency of the known drug-target interaction process $\varepsilon_{\mathrm{ij}}$ (known solution). The term $\mathrm{f}\left(\varepsilon_{\mathrm{ij}}\right)_{\text {ref }}=1$ if we already know from previous 
Table 1. Results of statistical analysis of the parameters of the flow cytometer showed significant differences between different concentrations and control groups.

\begin{tabular}{|c|c|c|c|c|}
\hline \multirow[b]{2}{*}{ Group1 } & & \multicolumn{2}{|c|}{ Group2 } & \\
\hline & & NC & DMSO & \\
\hline \multirow[t]{3}{*}{ Conc $(\mu \mathrm{g} / \mathrm{mL})$} & Parameters & $\mathrm{p}$ & $\mathrm{p}$ & MFI \\
\hline & SCC-H+ FSC-H+ & 0.872700 & 0.901186 & $494.46 \pm 43.85$ \\
\hline & $\mathrm{SCC}-\mathrm{H}+\mathrm{CD} 3 \mathrm{FITC}+$ & $0.041591^{\mathrm{a}}$ & 0.696366 & $171.51 \pm 27.08$ \\
\hline \multirow[t]{8}{*}{10} & $\mathrm{SCC}-\mathrm{H}+7-\mathrm{AAD}+$ & 0.819773 & 0.967863 & $233.2 \pm 38.38$ \\
\hline & SCC-H+ CD19bio-APC+ & 0.208782 & 0.508540 & $196.28 \pm 31.79$ \\
\hline & $\mathrm{CD} 3 \mathrm{FITC}+7 \mathrm{AAD}+$ & 0.130045 & 0.919263 & $105.5 \pm 17.82$ \\
\hline & CD3FITC + CD19bioAPC + & 0.083138 & 0.837216 & $37.77 \pm 37.77$ \\
\hline & CD19bioAPC+7AAD + & 0.061232 & 0.532019 & $114.96 \pm 38.58$ \\
\hline & CD19bioAPC+7AAD- & $0.035743^{\mathrm{a}}$ & 0.079477 & $59.18 \pm 21.09$ \\
\hline & SCC-H+ FSC-H+ & 0.574506 & 0.618669 & $480.7 \pm 43.34$ \\
\hline & $\mathrm{SCC}-\mathrm{H}+\mathrm{CD} 3 \mathrm{FITC}+$ & $0.009396^{\mathrm{a}}$ & 0.266945 & $165.3 \pm 26.80$ \\
\hline \multirow[t]{8}{*}{8} & $\mathrm{SCC}-\mathrm{H}+7-\mathrm{AAD}^{+}$ & 0.804872 & 0.962317 & $207.7 \pm 35.39$ \\
\hline & SCC-H+ CD19bio-APC+ & 0.080902 & 0.237483 & $197.6 \pm 122.9$ \\
\hline & $\mathrm{CD} 3 \mathrm{FITC}+7 \mathrm{AAD}+$ & $0.046746^{\mathrm{a}}$ & 0.633704 & $110.1 \pm 18.43$ \\
\hline & CD3FITC + CD19bioAPC + & $0.007041^{\mathrm{a}}$ & 0.172750 & $46.01 \pm 46.01$ \\
\hline & CD19bioAPC+7AAD + & 0.174550 & 0.835663 & $109.44 \pm 65.7$ \\
\hline & CD19bioAPC+7AAD- & 0.126061 & 0.256566 & $48.68 \pm 10.73$ \\
\hline & $\mathrm{SCC}-\mathrm{H}+\mathrm{FSC}-\mathrm{H}+$ & 0.271318 & 0.306663 & $358.43 \pm 31.67$ \\
\hline & $\mathrm{SCC}-\mathrm{H}+\mathrm{CD} 3 \mathrm{FITC}+$ & $0.023197^{\mathrm{a}}$ & 0.660648 & $212.41 \pm 15.51$ \\
\hline \multirow[t]{8}{*}{6} & $\mathrm{SCC}-\mathrm{H}+7-\mathrm{AAD}^{+}$ & 0.816395 & 0.976136 & $151.2 \pm 22.92$ \\
\hline & SCC-H+ CD19bio-APC+ & 0.100570 & 0.323846 & $131.83 \pm 18.23$ \\
\hline & CD3FITC $+7 \mathrm{AAD}+$ & 0.086465 & 0.917597 & $76.42 \pm 11.36$ \\
\hline & CD3FITC + CD19bioAPC + & $0.023535^{\mathrm{a}}$ & 0.583148 & $31.85 \pm 31.85$ \\
\hline & CD19bioAPC+7AAD + & $0.030059^{\mathrm{a}}$ & 0.418862 & $80.70 \pm 13.96$ \\
\hline & CD19bioAPC+7AAD- & $0.017667^{\mathrm{a}}$ & $0.044506^{\mathrm{a}}$ & $34.94 \pm 7.87$ \\
\hline & $\mathrm{SCC}-\mathrm{H}+\mathrm{FSC}-\mathrm{H}+$ & 0.421978 & 0.468035 & $362.12 \pm 30.54$ \\
\hline & $\mathrm{SCC}-\mathrm{H}+\mathrm{CD} 3 \mathrm{FITC}+$ & $0.024891^{\mathrm{a}}$ & 0.683118 & $121.69 \pm 16.33$ \\
\hline \multirow[t]{8}{*}{4} & $\mathrm{SCC}-\mathrm{H}+7-\mathrm{AAD}+$ & 0.894592 & 0.993257 & $164.9 \pm 22.86$ \\
\hline & SCC-H+ CD19bio-APC+ & 0.755928 & 0.988748 & $91.53 \pm 11.82$ \\
\hline & CD3FITC $+7 \mathrm{AAD}+$ & $0.032853^{\mathrm{a}}$ & 0.656848 & $68.41 \pm 10.94$ \\
\hline & CD3FITC + CD19bioAPC + & $0.049169^{\mathrm{a}}$ & 0.810848 & $28.13 \pm 28.13$ \\
\hline & CD19bioAPC+7AAD + & $0.034993^{\mathrm{a}}$ & 0.463901 & $63.18 \pm 22.05$ \\
\hline & CD19bioAPC+7AAD- & $0.016652^{\mathrm{a}}$ & $0.041979^{\mathrm{a}}$ & $31.54 \pm 7.23$ \\
\hline & $\mathrm{SCC}-\mathrm{H}+\mathrm{FSC}-\mathrm{H}+$ & 0.626131 & 0.675905 & $361.65 \pm 34.73$ \\
\hline & $\mathrm{SCC}-\mathrm{H}+\mathrm{CD} 3 \mathrm{FITC}+$ & $0.021791^{\mathrm{a}}$ & 0.640636 & $125.03 \pm 18.13$ \\
\hline
\end{tabular}

\footnotetext{
${ }^{a}$ Significant differences. $\mathrm{NC}$ is control negative and DMSO is control vehicle.
} 


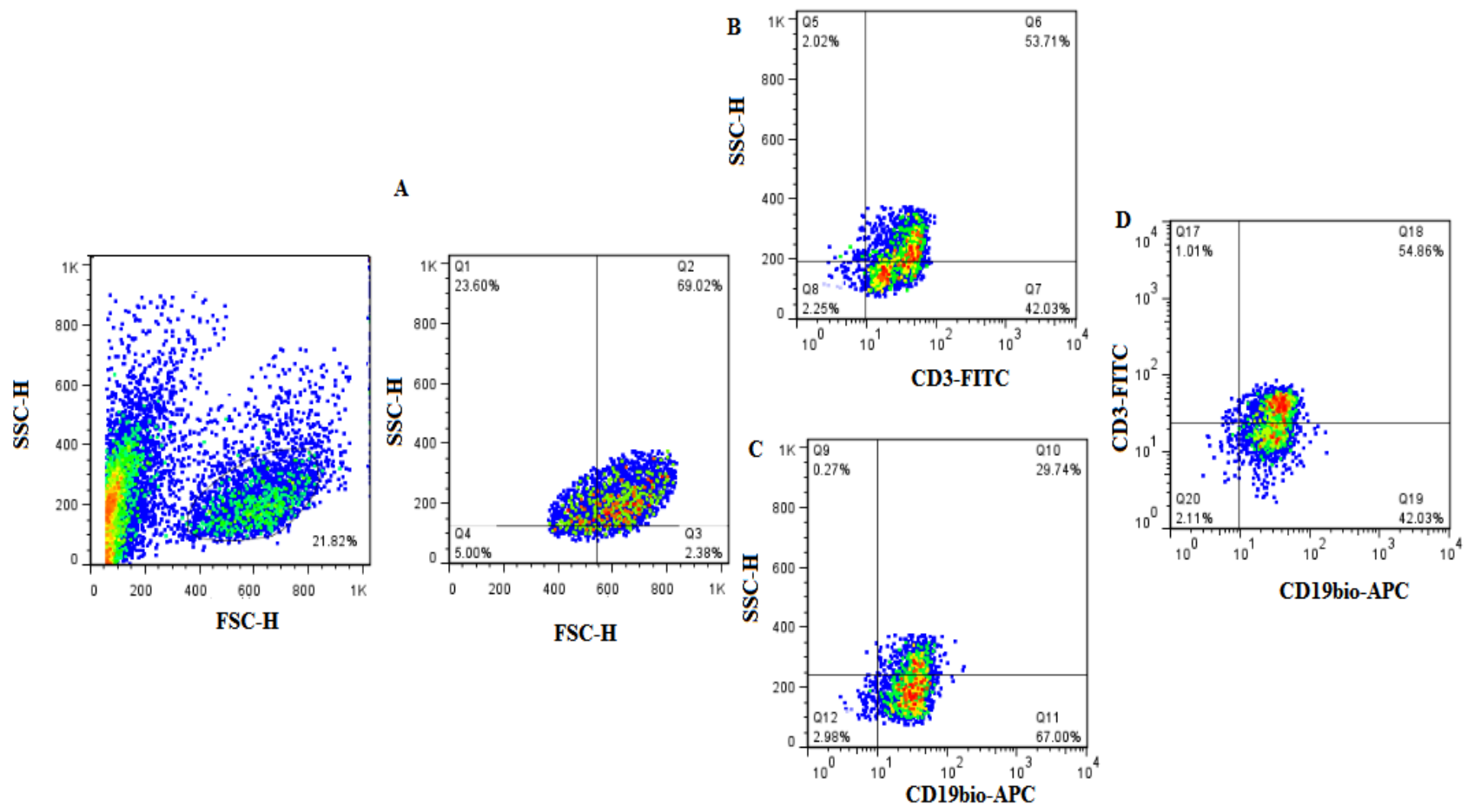

Fig. (2). Graphic type Pseudo-color of gating strategy for thymus B Lymphocytes of Balb/ C mouse analysis exposed to compound Dermofural at $10 \mu \mathrm{g} / \mathrm{mL}$. Example Total population Cell SSC-H \& FSC-H (A) lymphocytes marked with CD3-FITC (B) CD19bio-APC (C) and Both (D).

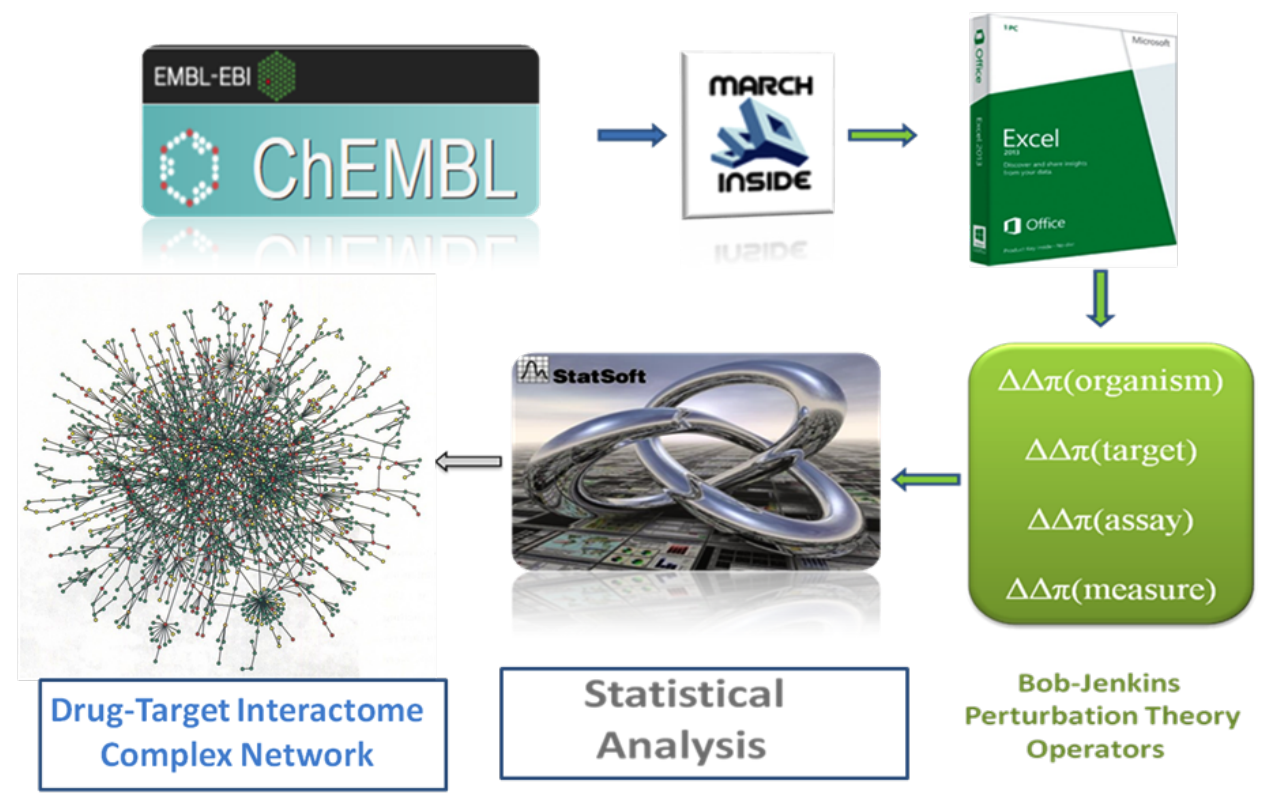

Fig. (3). Workflow for multi-target prediction of drug-target interactome networks.

experiments that the compound $\mathrm{i}^{\text {th }}$ interact with the cellular target in the boundary conditions $\mathrm{c}_{\mathrm{j}}, \mathrm{f}\left(\varepsilon_{\mathrm{ij}}\right)_{\text {ref }}=0$ otherwise. The atomic-bond polarization properties are the $\mathrm{Q}_{0}=$ atomic charge $(\mathrm{q}), \mathrm{Q}_{1}=$ Bond dipole moment $(\mathrm{D})$, and $\mathrm{Q}_{3}=$ atomic polarizability $(\alpha)$. The conditions of assay are the following $\mathrm{c}_{0}=$ chemical structure, $\mathrm{c}_{1}=$ biological property, $c_{2}=$ organism, and $c_{3}=$ molecular or cellular target.

In Table 2, we depict the classification matrix and the values of Ac, Sp, and Sn for training and validation series. This values are very good taking into consideration the results published for other problems with similar methodologies based on average models [32, 33] or perturbation models [29].

\section{CONCLUSION}

QSPR-perturbation theory using spectral moments have a high potential to develop different methods for the study of complex biosystems. Develop general models based on spectral moments to predict the effect of multiple input-output perturbations in complex systems it is possible. This new QSPR-Perturbation model can be 
Table 2. Results of QSPR-Perturbation model of drug-target interactome developed in this work.

\begin{tabular}{|c|c|c|c|c|c|c|}
\hline Order & Observed & Predicted & Values & \multicolumn{3}{|c|}{ Predicted Sets } \\
\hline $\mathbf{n}$ & sets & Statistics & $\%$ & $\mathbf{n}$ & $\mathbf{0}$ & 1 \\
\hline \multirow[t]{7}{*}{1} & 0 & $\mathrm{Sp}$ & 80.0 & 24814 & 19861 & 4953 \\
\hline & 1 & $\mathrm{Sn}$ & 82.3 & 17901 & 3171 & 14730 \\
\hline & Train $(t)$ & Ac & 81.0 & 42715 & & \\
\hline & 0 & $\mathrm{Sp}$ & 80.3 & 8233 & 6608 & 1625 \\
\hline & 1 & $\mathrm{Sn}$ & 82.8 & 6007 & 1034 & 4973 \\
\hline & Validation (v) & Ac & 81.3 & 14240 & & \\
\hline & Total $\mathrm{t}+\mathrm{v}$ & Ac & 81.1 & 56955 & & \\
\hline
\end{tabular}

${ }^{a}$ The positive (1) and negative control cases ( 0 ) were assigned as follows $L_{i j}=1 \Rightarrow z_{i j}>0=>v_{j}>\operatorname{Avg}\left(v_{j}\right)$ otherwise $L_{i j}=0$.

used to study properties like interaction of drugs with multiple targets or very different conditions, useful to predict changes on the ability of organic compounds to form complex interaction networks with molecular and cellular lymphocyte targets such as Dermofural.

\section{SUPPLEMENTARY MATERIAL}

Supplementary material is available on the publishers Web site along with the published article.

\section{CONFLICT OF INTEREST}

The authors confirm that this article content has no conflict of interest.

\section{ACKNOWLEDGEMENTS}

The authors thank the sponsorship of grant DCS-UQROO PROFOCIE (P/PROFOCIE-2014-23MSU0140Z-09 DCS) and research project CTQ2013-41229-P funded by MINECO, Spain.

\section{REFERENCES}

[1] Klein AB, Witonsky SG, Ahmed SA, et al. Impact of different cell isolation techniques on lymphocyte viability and function. J Immunoassay Immunochem 2006; 27: 61-76.

[2] Babic D, Graovac A, Gutman I. On a resonance energy model based on expansion in terms of acyclic moments: Exact results. Theoretica chimica acta 2013; 79: 403-411.

[3] Y. J, H. Z. Stability and reactivities based on moment analysis. Theoretica chimica acta 2013; 75: 279-297.

[4] Gutman I, Rucker C, Rucker G. On walks in molecular graphs. J Chem Inf Comput Sci 2001; 41: 739-45.

[5] Estrada E. On the topological sub-structural molecular design (TOSS-MODE) in QSPR/QSAR and drug design research. SAR QSAR Environ Res 2000; 11: 55-73.

[6] Estrada E, Gutierrez Y, González H. Modeling diamagnetic and magnetooptic properties of organic compounds with the TOSSMODE approach. J Chem Inf Comput Sci 2000; 40: 1386-1399.

[7] Estrada E, Uriarte E. Quantitative structure--toxicity relationships using TOPS-MODE. 1. Nitrobenzene toxicity to Tetrahymena pyriformis. SAR QSAR Environ Res 2001; 12: 309-24.

[8] Estrada E, Molina E, Uriarte E. Quantitative structure-toxicity relationships using TOPS-MODE. 2. Neurotoxicity of a noncongeneric series of solvents. SAR QSAR Environ Res 2001; 12: 445-59.

[9] Estrada E, Uriarte E, Gutierrez Y, Gonzalez H. Quantitative structure-toxicity relationships using TOPS-MODE. 3. Structural factors influencing the permeability of commercial solvents through living human skin. SAR QSAR Environ Res 2003; 14: 145-63.

[10] Estrada E, Peña A, García-Domenech R. Designing sedative/hypnotic compounds from a novel substructural graphtheoretical approach. J Comput Aided Mol Des 1998; 12: 583-95.
[11] Estrada E, Peña A. In silico studies for the rational discovery of anticonvulsant compounds. Bioorg Med Chem 2000; 8: 2755-70.

[12] Gonzalez-Diaz H, Vilar S, Santana L, Uriarte E. Medicinal chemistry and bioinformatics - Current trends in drugs discovery with networks topological indices. Current Topics in Medicinal Chemistry 2007; 7: 1015-1029.

[13] Gonzalez-Diaz H, Prado-Prado F, Ubeira FM. Predicting antimicrobial drugs and targets with the MARCH-INSIDE approach. Curr Top Med Chem 2008; 8: 1676-90.

[14] Duardo-Sanchez A, Munteanu CR, Riera-Fernández P, López-Díaz A, Pazos A, González-Díaz H. Modelling complex metabolic reactions, ecological systems, and financial-legal networks with MIANN models based on Markov-Wiener centralities. J Chemical Information and Modelling 2013: submitted.

[15] Gonzalez-Diaz H. Bioinformatics and Quantitative StructureProperty Relationship (QSPR) Models. Current Bioinformatics 2013; 8: 387-389.

[16] Gonzalez-Diaz H, Riera-Fernandez P, Pazos A, Munteanu CR. The Rucker-Markov invariants of complex Bio-Systems: Applications in Parasitology and Neuroinformatics. Biosystems 2013; 111: 199207.

[17] Riera-Fernandez P, Munteanu CR, Martin-Romalde R, DuardoSanchez A, Gonzalez-Diaz H. Markov-Randic Indices for QSPR Re-Evaluation of Metabolic, Parasite-Host, Fasciolosis Spreading, Brain Cortex and Legal-Social Complex Networks. Current Bioinformatics 2013; 8: 401-415.

[18] Box GEP, Jenkins GM. Time series analysis. Holden-Day 1970.

[19] Luan F, Cordeiro MN, Alonso N, et al. TOPS-MODE model of multiplexing neuroprotective effects of drugs and experimentaltheoretic study of new 1,3-rasagiline derivatives potentially useful in neurodegenerative diseases. Bioorg Med Chem 2013; 21: 18709.

[20] Mingoia RT. NDL, Ching-Hui Yang, Xing Han. Primary culture of rat hepatocytes in 96-well plates: Effects of extracellular matrix conWguration on cytochrome P450 enzyme activity and inducibility, and its application in in vitro cytotoxicity screening. Toxicology in Vitro 2007; 21: 165-173.

[21] Weyermann J. LD, Zimmer Andreas... A practical note on the use of cytotoxicity assays. International J Pharmaceutics 2005; 288: 369-376.

[22] Tenorio-Borroto E, Penuelas Rivas CG, Vasquez Chagoyan JC, et al. ANN multiplexing model of drugs effect on macrophages; theoretical and flow cytometry study on the cytotoxicity of the antimicrobial drug G1 in spleen. Bioorg Med Chem 2012; 20: 6181-94.

[23] Cao LF KL, Tran V, Mi S, Jensen MC, Blanchard S, Kalos M Development and application of a multiplexable flow cytometrybased assay to quantify cell-mediated cytolysis. Cytometry A 2010; 77: 534-45.

[24] Riss TL MR. Use of multiple assay endpoints to investigate the effects of incubation time, dose of toxin, and plating density in cellbased cytotoxicity assays. Assay Drug Dev Technol 2004; 2: 5162 
[25] Marqués-Gallego P, den Dulk H, Backendorf C, Brouwer J, Reedijk J, Burke JF. Accurate non-invasive image-based cytotoxicity assays for cultured cells. BMC Biotechnol 2010; 10: 43.

[26] Sekhon BK, Roubin RH, Tan A, Chan WK, Sze DM. Highthroughput screening platform for anticancer therapeutic drug cytotoxicity. Assay Drug Dev Technol 2008; 6: 711-21.

[27] Perez Machado Giselle. GBJI, Castañedo N, Creus A, Marcos R. In vitro genotoxicity testing of the furylethylene derivative UC-245 in human cells. Mutagenesis 2004; 19: 75-80.

[28] OECD ED. Guidance document on using cytotoxicity tests to estimate starting doses for acute oral systemic toxicity tests. OECD Series on Testing and Assessment 2010; 20: 1-54.

[29] Gonzalez-Diaz H, Arrasate S, Gomez-San Juan A, et al. New theory for multiple input-output perturbations in complex molecular systems. 1. Linear QSPR electronegativity models in physical, organic, and medicinal chemistry. Curr Top Med Chem 2013.
[30] González-Díaz H, Pérez-Montoto LG, Ubeira FM. Model for vaccine design by prediction of B-epitopes of IEDB given perturbations in peptide sequence, in vivo process, experimental techniques, and source or host organisms. Clin Develop Immunol 2014; 2014: $1-20$.

[31] Gaulton A, Bellis LJ, Bento AP, et al. ChEMBL: a large-scale bioactivity database for drug discovery. Nucleic Acids Research 2012; 40: D1100-7.

[32] Speck-Planche A, Kleandrova VV, Luan F, Cordeiro MN. In silico discovery and virtual screening of multi-target inhibitors for proteins in Mycobacterium tuberculosis. Comb Chem High Throughput Screen 2012; 15: 666-73.

[33] Speck-Planche A, Kleandrova VV, Luan F, Cordeiro MN Chemoinformatics in anti-cancer chemotherapy: multi-target QSAR model for the in silico discovery of anti-breast cancer agents. Eur J Pharm Sci 2012; 47: 273-9. 